\title{
人纤维蛋白原与聚四氟乙烯表面间 相互作用的研究*
}

\author{
张汉炏、张秀芳公衍道 赵南明 \\ (清华大学生命科学与工程研究院, 生物膜与膜生物工程国家重点实验室, 北京 100084)
}

\section{关键词人纤维蛋白原 聚四雨乙烯 吸附 二级结构 界面}

在生物医学材料的研究和应用中, 亟待解决的一个重要问题是这些材料的血液相容性问 题. 一般认为生物医学材料表面吸附了纤维蛋白原 (Human fibrinogen, $\mathrm{Fg}$ ), $\gamma$ - 球蛋白等糖 蛋白后, 就会与血小板形成复合体而粘附于材料表面, 加速内源性凝血, 促使溶胶状态的纤维 蛋白原转变成为凝胶状态的纤维蛋白, 从而造成凝血. 尽管人们对于血浆蛋白与各种材料表面 的相互作用已进行了大量的研究, 然而血浆蛋白在材料表面的吸附机理至今尚不清楚 ${ }^{[1]}$. 为 了搞清楚材料表面凝血的机理, 首先需要从界面反应的角度研究吸附在材料表面的血浆蛋白 分子的结构和功能上发生的变化. Fg 是理解凝血、抗凝、血栓形成与溶栓机制的核心 ${ }^{[2]}$ 本文 将 Fg 吸附在平均粒径约 $100 \mu \mathrm{m}$ 不透明的聚四氟乙烯 (Poly tetrafluro ethylene, PTFE) 微粒 表面, 用圆二色 (Circular dichroism, CD) 光谱方法研究它们间的相互作用, 为从分子水平理 解 PTFE 表面的血液相容性提供了一定的依据.

\section{1 材料与方法}

Fg 由中国药品生物制品检定所血液制品室生产, PTFE 微粒由清华大学化工系高分子材 料室提供.

用 PBS (pH 7.0) 缓冲液将 $\mathrm{Fg}$ 配制成浓度分别为 1,2 和 $3 \mathrm{mg} / \mathrm{mL}$ 的溶液, $4{ }^{\circ} \mathrm{C}$ 摚拌 $1 \mathrm{~h}, 10^{\circ} \mathrm{C}$ 静置 $12 \mathrm{~h}$, 简称为原液, 作为对照组. 在室温 (约 $23{ }^{\circ} \mathrm{C}$ ) 条件下, 用 $\mathrm{J}-500 \mathrm{C}$ 分光偏振计测其 CD 谱. 取 3 个小烧杯, 每个装 $1 \mathrm{~g}$ 的 PTFE 微粒, 加人 $2 \mathrm{~mL}$ 上述不同浓度 的 $\mathrm{Fg}$ 蛋白溶液, $4{ }^{\circ} \mathrm{C}$ 摚拌 $1 \mathrm{~h}$ 充分分散, $10^{\circ} \mathrm{C}$ 静置吸附 $12 \mathrm{~h}$, 所得浑浊液称为浊液, 测量浊液 组 CD 谱, 此处必须保证不同浓度的浊液在通光范围内的 PTFE 微粒的粒子密度相同. 将 3 种 不同浓度的浊液离心, 取上清, 称为清液, 测量清液组的 CD 谱.

取 3 支试管, 每支放人 $1 \mathrm{~g}$ 的 PTFE 微粒, 均加 $2 \mathrm{~mL}$ 浓度为 $2 \mathrm{mg} / \mathrm{mL}$ 的 $\mathrm{Fg}$ 溶液, 室温摚 拌 $10 \mathrm{~min}$ 后, 分别静置 10,30 和 $70 \mathrm{~min}$, 即吸附时间依次为 20,40 和 $80 \mathrm{~min}$, 用自制装 置立即除去液体和未被吸附的 Fg 分子, 所得吸附有 Fg 分子的 PTFE 干燥微粒都取 $0.2 \mathrm{~g}$, 分别放人盛有 $2 \mathrm{~mL}$ PBS 缓冲液的试管中; 振荡充分分散, $10{ }^{\circ} \mathrm{C}$ 脱附 $12 \mathrm{~h}$ 后所得溶液称 
为脱附液. $280 \mathrm{~nm}$ 测吸收率. 用标准浓度曲线求浓度, 并测 $\mathrm{CD}$ 谱. 原液、清液和脱附液的 CD 谱均用本实验室所编的 CDD32 计算机程序进行定量分析.

\section{2 结果与讨论}

(1) 由图 1 可见, 不同浓度的 $\mathrm{Fg}$ 原液的 CD 谱, 均在 $208 \mathrm{~nm}$ 处有一明显负峰, 与它们相对应 的清液的 CD 谱 (图 2) 也有类似的特征, 定量分析的结果列于表 1 和表 2 , 说明清液的二级结构 和原液的相同. 可以认为清液中虽然含有一些脱附蛋白, 但经过 $12 \mathrm{~h}$ 的长时间吸附, 在吸附 脱附达到平衡后, 它们并不是直接从 PTFE 微粒的表面上脱附下来的, 而是从吸附在 PTFE 微 粒表面的 Fg 分子层的外表面上脱附下来的, 它们的结构变化不大, 脱附后易于恢复.

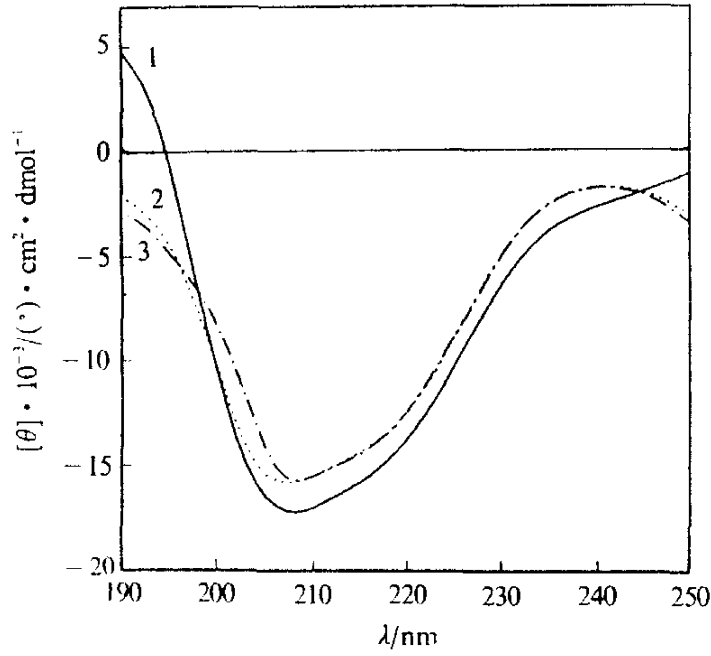

图 $1 \mathrm{Fg}$ 原液的 CD 谱

1 一浓度为 $1 \mathrm{mg} / \mathrm{mL}, 2$ 一浓度为 $2 \mathrm{mg} / \mathrm{mL}, 3-$ 浓度为 $3 \mathrm{mg} / \mathrm{mL}$

表 1 原液中 $\mathrm{Fg}$ 分子的二级结构

\begin{tabular}{cccc}
\hline $\begin{array}{c}\mathrm{Fg} \text { 浓度/ } \\
\mathrm{mg} \cdot \mathrm{mL}^{-1}\end{array}$ & $\begin{array}{c}\alpha-\text { 螺旋/ } \\
\%\end{array}$ & $\begin{array}{c}\beta-\text { 折叠/ } \\
\%\end{array}$ & $\begin{array}{c}\text { 无规卷曲/ } \\
\%\end{array}$ \\
\hline 1 & 19.17 & 27.50 & 53.33 \\
2 & 18.21 & 28.86 & 52.93 \\
3 & 19.22 & 30.17 & 50.61 \\
平均 & 18.87 & 28.84 & 52.29 \\
\hline
\end{tabular}

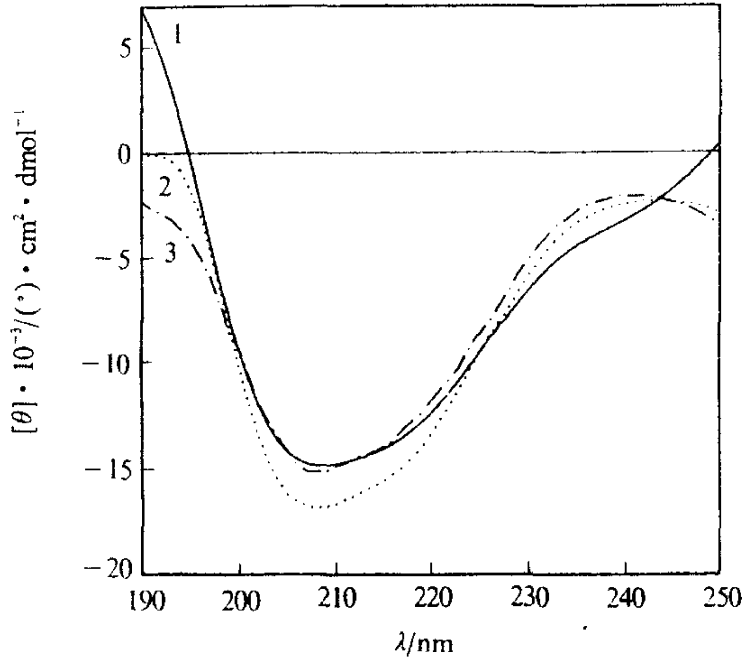

图 $2 \mathrm{Fg}$ 清液的 CD 谱 1 - 浓度为 $0.6 \mathrm{mg} / \mathrm{mL}, 2$ 一浓度为 $1.4 \mathrm{mg} / \mathrm{mL}$, 3 一浓度为 $2.2 \mathrm{mg} / \mathrm{mL}$

表 2 清液中 $\mathrm{Fg}$ 分子的二级结构

\begin{tabular}{cccc}
\hline $\begin{array}{c}\mathrm{Fg} \text { 浓度/ } \\
\mathrm{mg} \cdot \mathrm{mL}^{-1}\end{array}$ & $\begin{array}{c}\alpha-\text { 螺旋/ } \\
\%\end{array}$ & $\begin{array}{c}\beta-\text { 折舊/ } \\
\%\end{array}$ & $\begin{array}{c}\text { 无规卷曲/ } \\
\%\end{array}$ \\
\hline 0.6 & 19.85 & 26.09 & 54.06 \\
1.4 & 18.43 & 28.69 & 52.88 \\
2.2 & 18.42 & 29.43 & 52.15 \\
平均 & 18.90 & 28.07 & 53.03 \\
\hline
\end{tabular}

(2) 与原液相对应的浊液组的 CD 谱 (图 3)表明,相对于 $208 \mathrm{~nm}$ 的位置, 负峰明显发生红 移,红移的大小分别为 2,4 和 $6 \mathrm{~nm}$, 说明随浓度的升高红移增大. 由于清液的二级结构未发 生变化, 所以对红移无贡献, 又因为在 3 种浓度的浊液中 PTFE 微粒的粒子密度相同, 从而使 PTFE 微粒因散射等原因对 CD 谱的影响相同. 在 $250 \sim 200 \mathrm{~nm}$ 范围内 Fg 的吸收显著, 这样 在光强一定的条件下, 随浓度的升高吸收增强, 透射和散射的比例降低, 使得由散射引起的红 移减小, 故可以认为随浓度升高浊液 CD 谱的红移增大是由于吸附于 PTFE 微粒表面的 Fg 分 
子二级结构发生了变化, 并且随浓度升高这种结构发生变化的 Fg 分子在 PTFE 微粒表面的 吸附量增大所导致的.

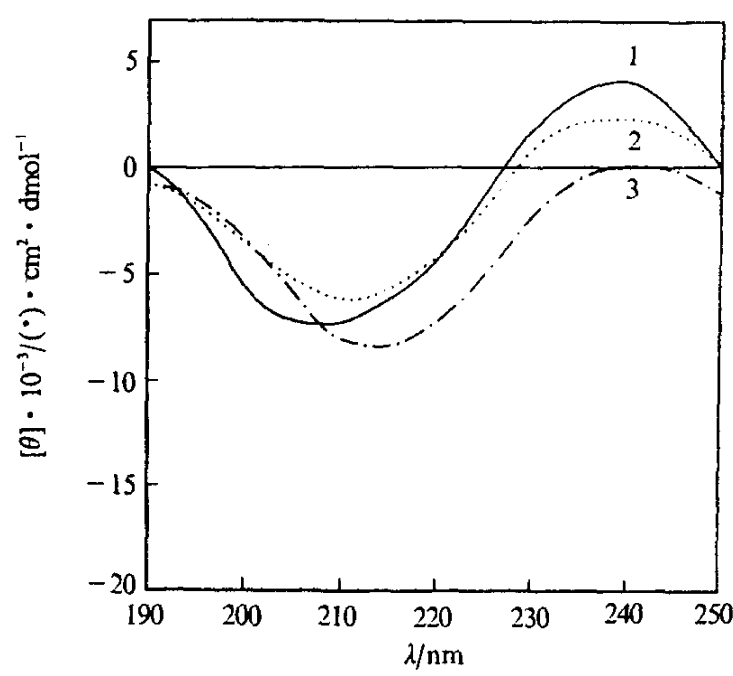

图 $3 \mathrm{Fg}$ 与 PTFE 浑浊液的 CD 谱 1 - $\mathrm{Fg}$ 浓度为 $1 \mathrm{mg} / \mathrm{mL}, 2-\mathrm{Fg}$ 浓度为 $2 \mathrm{mg} / \mathrm{mL}$, 3 - $\mathrm{Fg}$ 浓度为 $3 \mathrm{mg} / \mathrm{mL}$

表 3 由吸附有 Fg 的 PTFE 表面脱附 下来的 $\mathrm{Fg}$ 分子的二级结构

\begin{tabular}{ccccc}
\hline $\begin{array}{c}\text { 脱附液浓度/ } \\
\mathrm{mg} \cdot \mathrm{mL}^{-1}\end{array}$ & $\begin{array}{c}\text { 吸附时间/ } \\
\min \end{array}$ & $\begin{array}{c}\alpha \text {-螺旋/ } \\
\%\end{array}$ & $\begin{array}{c}\beta \text {-折叠/ } \\
\%\end{array}$ & $\begin{array}{c}\text { 无规卷曲/ } \\
\%\end{array}$ \\
\hline 0.15 & 20 & 20.13 & 34.54 & 45.15 \\
0.09 & 40 & 17.64 & 40.82 & 41.54 \\
0.05 & 80 & 12.82 & 39.14 & 48.04 \\
\hline
\end{tabular}

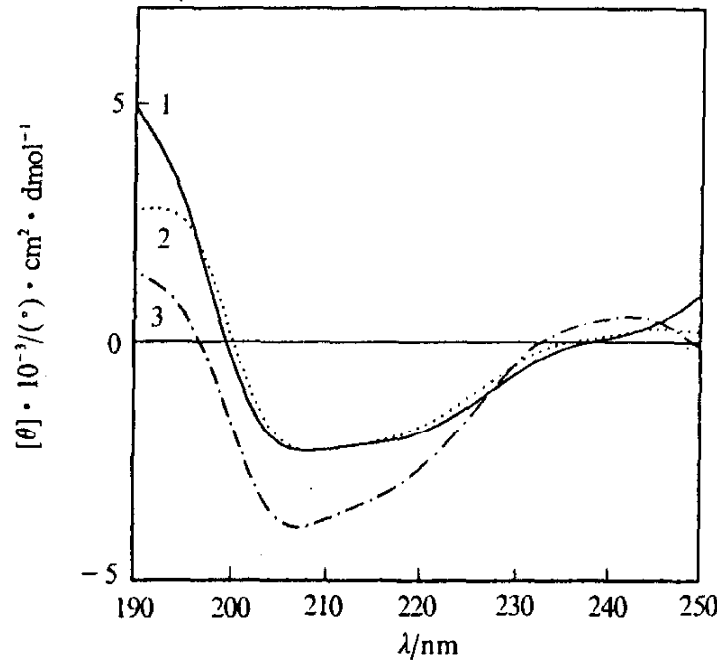

图 $4 \mathrm{Fg}$ 脱附液的 $\mathrm{CD}$ 谱

1 一浓度为 $0.15 \mathrm{mg} / \mathrm{mL}, 2$ - 浓度为 $0.09 \mathrm{mg} / \mathrm{mL}$, 3 - 浓度为 $0.05 \mathrm{mg} / \mathrm{mL}$,

屏状发卡连串

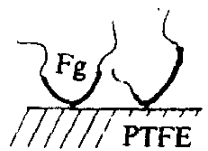

(a) 初期

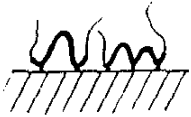

(b) 中期

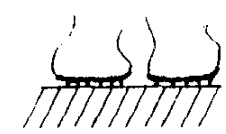

(c)末期
图 $5 \mathrm{Fg}$ 吸附于 PTFE 表面的模型

(3) 如图 4 所示, 脱附液 CD 谱的明显负峰的峰位分别在 208.5, 207.5 和 $206 \mathrm{~nm}$ 处, 都偏离了 $208 \mathrm{~nm}$ 处的原液峰位, 由表 3 所示的定量分析结果可知, 吸附时间从 $20 \mathrm{~min}$ 增 加到 $80 \mathrm{~min}$, 脱附后 $\mathrm{Fg}$ 分子的 $\beta$ - 折叠和无规卷曲结构的含量增加, $\alpha$-螺旋明显减少, 说明在 没有达到吸附 - 脱附平衡时, 吸附时间不同, Fg 分子的 $\alpha$ - 螺旋结构因受 PTFE 表面作用而被 破坏的程度不同, 同时说明脱附的 Fg 分子发生了不可逆的二级结构变化.

(4) 已有研究结果表明材料表面吸附的蛋白质的二级结构会发生变化 ${ }^{[3]}$, 材料的疏水性对 $\mathrm{Fg}$ 构象的改变起重要作用, 随疏水性的增强, 材料表面对 $\mathrm{Fg}$ 构象的改变增大 ${ }^{[4]}$. 疏水性极强 的 PTFE 表面对 Fg 结构的改变显著, 疏水力起了重要作用. 依据 $\mathrm{Fg}$ 分子结构及氨基酸的性 质可知, $\mathrm{Fg}$ 分子在 3 股超螺旋结构域内间歇分布着非极性氨基酸, 它们都朝向螺旋内部, 而极 性氨基酸都朝向螺旋外部, 该结构域基本由 $\alpha$-螺旋组成. CD 谱结果表明, 在达到吸附 - 解附 平衡前, 随时间的增加 Fg 分子的 $\alpha$ - 螺旋减少, 认为是一部分非极性氨基酸与 PTFE 表面依靠 疏水力结合, 使更多的非极性氨基酸露出 $\mathrm{Fg}$ 分子表面所导致的结果. 又因随时间的增加, 
脱附液的浓度减小 (见表 3), 这表明从 PTFE 表面脱附下来的 $\mathrm{Fg}$ 分子减少, 说明 $\mathrm{Fg}$ 分子与 PTFE 表面间的结合力增大, 所以认为随时间的增加 Fg 分子的疏水部位与 PTFE 表面依靠疏 水力结合的几率增大, 结合位点增多. 我们把三球棒状的 $\mathrm{Fg}$ 分子 ${ }^{[\mathrm{g}}$ 简化为线状, 综合本研究 结果将 Fg 分子在 PTFE 表面的吸附过程分为初期、中期和末期, 提出如下模型：在吸附初 期, Fg 分子与 PTFE 表面间主要依靠疏水力形成 1 个结合位点, Fg 分子在结合后形成尾状 (tail) 形态, Fg 分子结构的变化最小, 与 PTFE 表面间的结合力最弱, 易于脱附下来; 在吸附 中期, 由于 Fg 分子与 PTFE 表面间相互作用的时间增长, Fg 分子表面的疏水部位与 PTFE 表面 间依靠疏水力结合的概率增大, Fg 分子与 PTFE 表面的结合位点增加到 2 个或 2 个以上, 形 成发卡 (loop) 形态, Fg 分子结构变化增大, 与 PTFE 表面的结合力增强, 较难脱附下来; 在吸 附末期, 由于 Fg 分子与 PTFE 表面间相互作用的时间进一步增长, Fg 分子表面的疏水部位 以及因与 PTFE 表面作用而露出表面的疏水部位都与 PTFE 表面结合, Fg 分子与 PTFE 表面 的结合位点进一步增多, 最终达到一定环境下的最大可能限度, 并且由于分子间碰撞、静电 力、范德瓦尔斯力等的协助作用使得原来的发卡拉直而形成连串 (train) 形态, $\mathrm{Fg}$ 分子的结构 变化最大, 与 PTFE 表面间的结合力最强, 很难脱附下来, 见图 5, 该模型尚需其它方法进一步证 实. 我们认为 Fg 分子与 PTFE 表面间相互作用的可能机理是： Fg 分子与 PTFE 表面间主要 依靠疏水力结合, 结合后 $\mathrm{Fg}$ 分子的部分 $\alpha$ - 螺旋结构受到破坏向 $\beta$ - 折叠和无规卷曲转变, 从而 露出更多的疏水部位与 PTFE 表面进一步结合, 直至达到一定环境下的最大可能限度的结 合.

\section{参 考 文 献}

1 Kondo Akahiko, Oku Shinya, Higashitani Ko. Structural changes in protein molecules absorbed on ultrafine silica particles. Journal of Colloid and Interface Science, 1991, 143(1): $214 \sim 221$

2 郝文学主编. 血栓与抗栓酶. 沈阳: 沈阳出版社, 1992. $25 \sim 36$

3 Andrade J D, Hlady V L, Van Wagene R A. Effects of plasma protein conformation and activity. Pure \& Appl Chem, 1984, 56(10): $1345 \sim 1350$

4 Lu D R, Park K. Effect of surface hydropobicity on the conformational changes of adsorbed fibrinogen. Journal of Colloid \& Interface Science, 1991, 144(1): 271 281

5 Beck E A, Furlan M. Structure \& function of fibrinogen. Current Problems in Clinical Biochemistry: 14, Variants of Human Fibrinogen. Huber, 1984, 38 58 